\title{
Anti-PD1/CTLA4 Bispecific Antibody XmAb20717
}

National Cancer Institute

\section{Source}

National Cancer Institute. Anti-PD1/CTLA4 Bispecific Antibody XmAb20717. NCI

Thesaurus. Code C150463.

A Fc-eng ineered bispecific antibody directed against the human negative

immunoregulatory checkpoint receptors programmed cell death protein 1 (PD-1; PDCD1;

CD279) and cytotoxic T-lymphocyte-associated antigen 4 (CT LA4; CT LA-4), with

potential immune checkpoint inhibitory and antineoplastic activities. Upon administration, anti-PD1/CT LA4 bispecific antibody XmAb20717 targets and binds to both PD-1 and CT LA4 expressed on tumor-infiltrating T-lymphocytes (TILS) and inhibits the PD-1- and CT LA4-mediated downregulation of T-cell activation and proliferation. This restores immune function and activates a cytotoxic T-lymphocyte (CT L)-mediated immune response against tumor cells. Both PD-1 and CT LA4 are selectively expressed on TILs in the tumor microenvironment (TME) and negatively regulate the activation and effector functions of T-cells. They play key roles in the downregulation of the immune system and tumor evasion from host immunity. Dual checkpoint blockade of PD1 and CT LA4 with XmAb20717 may enhance T cell activation and proliferation more than the blockade of either immune checkpoint receptor alone. The engineered Fc domain increases the stability and half-life of the antibody. 